Western University

Scholarship@Western

Chemistry Publications

Chemistry Department

Summer 8-10-2017

\title{
(Co)Polymers Containing Boron Difluoride 3-Cyanoformazanate Complexes: Emission Enhancement via Random Copolymerization
}

Joe Gilroy

jgilroy5@uwo.ca

Samantha Novoa

Follow this and additional works at: https://ir.lib.uwo.ca/chempub

Part of the Chemistry Commons

Citation of this paper:

Gilroy, Joe and Novoa, Samantha, "(Co)Polymers Containing Boron Difluoride 3-Cyanoformazanate Complexes: Emission Enhancement via Random Copolymerization" (2017). Chemistry Publications. 79.

https://ir.lib.uwo.ca/chempub/79 


\title{
Polymer Chemistry
}

\section{ARTICLE}

\section{(Co)Polymers Containing Boron Difluoride 3-Cyanoformazanate Complexes: Emission Enhancement via Random Copolymerization}

Received 00th January 20xx, Accepted 00th January 20xx DOI: $10.1039 / \times 0 \times x 00000 x$ www.rsc.org/

\author{
Samantha Novoa ${ }^{a}$ and Joe B. Gilroy*a
}

\begin{abstract}
Ring-opening metathesis polymerization was used to produce polymers bearing an asymmetrically substituted boron difluoride 3-cyanoformazanate complex. The polymers were found to retain many of the unique characteristics of molecular boron difluoride complexes of 3-cyanoformazanates, including intense light absorption at ca. $560 \mathrm{~nm}$ and reversible electrochemical reductions implicating the radical anion and dianion forms of the formazanate complexes in the repeating unit of the polymer backbone. The polymers were also found to be emissive, with emission maxima centred at ca. $665 \mathrm{~nm}$. The monomer employed in this study had a fluorescence quantum yield of $30 \%$, while homopolymers were weakly emissive and block copolymers were essentially non-emissive. The development of a monomer 'dilution' strategy, via random copolymerization, resulted in rejuvination of the emission at $c a .665 \mathrm{~nm}$ up to a maximum quantum yield of $24 \%$ when the mole fraction of the repeating units bearing boron difluoride 3 -cyanoformazanate complexes $\left(f_{B F 2 N}\right)$ was 0.08 .
\end{abstract}

\section{Introduction}

Polymers constructed from a diverse range of boroncontaining molecular materials ${ }^{1-4,5-9}$ have shown widespread utility in a variety of areas, including: spectroscopic sensing, ${ }^{10-}$ ${ }^{14}$ fluorescence imaging, ${ }^{15-18}$ redox-flow batteries, ${ }^{19}$ and lightharvesting applications. ${ }^{20-23}$ Amongst the most common examples of this subclass of polymeric materials are those that incorporate boron difluoride $\left(\mathrm{BF}_{2}\right)$ adducts of chelating oxygen and/or nitrogen donors. These polymers often exhibit unique and useful absorption, emission, and electrochemical properties. For example, Fraser and co-workers have demonstrated the ability to modulate solid-state fluorescence and phosphorescence of derivatives of polymer 1 by varying the length of appended poly(lactic acid) chains and incorporating heavy atoms. These findings ultimately allowed for the use of the polymers produced as tumour hypoxia imaging agents. ${ }^{24}$ The Chujo group synthesized a methacrylate derivative with a pendant boron-dipyrromethene (BODIPY) unit that was copolymerized with polystyrene using reversible addition fragmentation chain transfer (RAFT) polymerization to yield polymer $2 .^{25}$ This polymer self-assembled into nanosized particles that exhibited absorption and emission

Department of Chemistry and the Centre for Advanced Materials and Biomaterials Research (CAMBR), The University of Western Ontario, 1151 Richmond St. N., London, Ontario, Canada, N6A 5B7. Tel: +1-519-661-2111 ext. 81561:E-mail:joe.gilroy@uwo.ca

Electronic Supplementary Information (ESI) available: [NMR spectra; UV-vis absorption and emission spectra; cyclic voltammograms; thermal gravimetric analysis and gel permeation chromatography data]. See DOI: 10.1039/x0xx00000x characteristics similar to that of the free BODIPY monomer, although the quantum efficiency of the particles was increased relative to the corresponding monomer. Manners et al. have incorporated BODIPY units into polymers (e.g., 3) that form the corona of self-assembled block copolymer micelles allowing for the visualization of the unique morphologies produced and tracking of the solution-based crystallization-driven selfassembly process. ${ }^{26-28}$

The ring-opening metathesis polymerization (ROMP) of side-chain polymers bearing boron difluoride $\left(\mathrm{BF}_{2}\right)$ triaryl formazanate complexes $\mathbf{4}$ has been previously explored in detail. ${ }^{29}$ The redox-active polymers produced possessed many of the attractive traits of related molecular species, ${ }^{30,31}$

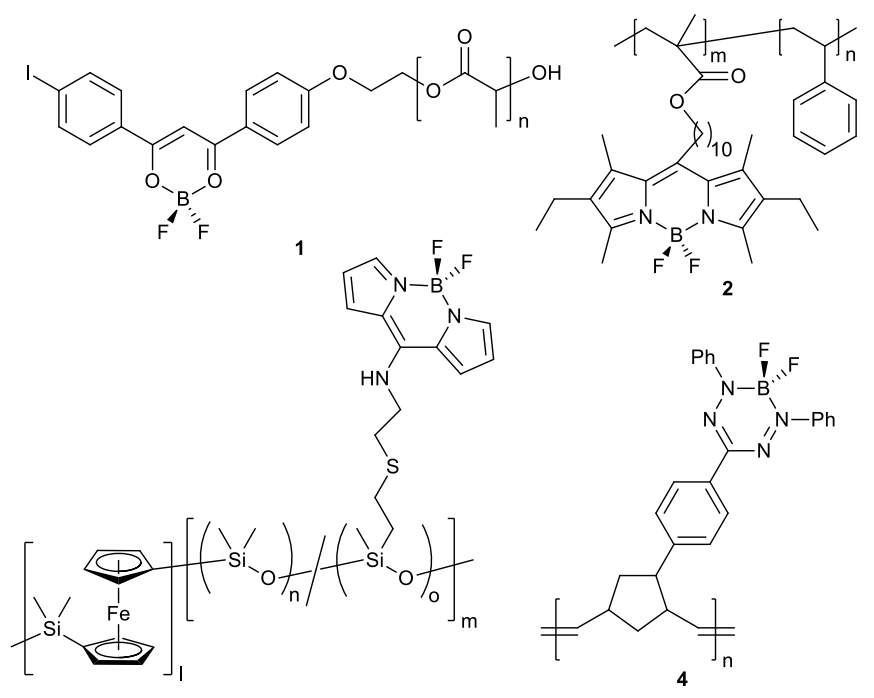

3

J. Name., 2013, 00,1-3 | 
although they were essentially non-emissive. In the current report, we set out to produce a new family of emissive polymers that may show utility in many of the applications highlighted above. Specifically, side-chain homopolymers along with random and block copolymers bearing pendant $\mathrm{BF}_{2}$ 3-cyanoformazanate complexes were targeted, as this subclass of molecular $\mathrm{BF}_{2}$ formazanate complexes has been shown to possess structurally tunable absorption, emission, and electrochemical properties. ${ }^{32-34}$ Furthermore, we thoroughly examined the effect of copolymerization on the photophysical properties of the polymers produced.

\section{Experimental Section}

\section{General Considerations}

Reactions and manipulations were carried out under a $\mathrm{N}_{2}$ atmosphere using standard Schlenk techniques unless otherwise stated. Solvents were obtained from Caledon Laboratories, dried using an Innovative Technologies Inc. solvent purification system, collected under vacuum, and stored under a nitrogen atmosphere over 4 Å molecular sieves. N3-N, ${ }^{35}$ HCC-BF2, ${ }^{36}$ DND, ${ }^{37}$ and the 3-bromopyridine derivative of Grubbs' third generation catalyst ${ }^{38}$ (G3) were synthesized according to reported procedures. All other reagents were purchased from Sigma Aldrich, Alfa Aesar or TCl America and used as received. NMR spectra were recorded on a $400 \mathrm{MHz}$ $\left({ }^{1} \mathrm{H}: 399.8 \mathrm{MHz},{ }^{11} \mathrm{~B}: 128.3 \mathrm{MHz},{ }^{13} \mathrm{C}\left\{{ }^{1} \mathrm{H}\right\}: 100.5 \mathrm{MHz},{ }^{19} \mathrm{~F}\right.$ : 376.1 MHz) or $600 \mathrm{MHz}\left({ }^{13} \mathrm{C}\left\{{ }^{1} \mathrm{H}\right\}\right.$ : $\left.150.7 \mathrm{MHz}\right)$ Varian INOVA instruments. ${ }^{1} \mathrm{H}$ NMR spectra were referenced to residual $\mathrm{CHCl}_{3}$ at $7.27 \mathrm{ppm}$ and ${ }^{13} \mathrm{C}\left\{{ }^{1} \mathrm{H}\right\} \mathrm{NMR}$ spectra were referenced to $\mathrm{CDCl}_{3}$ at $77.00 \mathrm{ppm} .{ }^{11} \mathrm{~B} \mathrm{NMR}$ spectra were referenced internally to $\mathrm{BF}_{3} \bullet \mathrm{OEt}_{2}$ at $0 \mathrm{ppm} .{ }^{19} \mathrm{~F} \mathrm{NMR}$ spectra were referenced internally to $\mathrm{CFCl}_{3}$ at $0 \mathrm{ppm}$. UV-vis absorption spectra were recorded using a Cary 5000 Scan instrument using standard quartz cells ( $1 \mathrm{~cm}$ path length) with a scan range of 200 to $700 \mathrm{~nm}$. The absorption response of this instrument is linear up to 8 absorption units. Emission spectra were recorded for degassed solutions using a Photon Technology International QM-4 SE Spectrofluorometer. The excitation wavelength was chosen as the wavelength of maximum absorption $\left(\lambda_{\max }\right)$ for the corresponding compounds. Excitation spectra were collected to verify the validity of this approach. Emission quantum yields were estimated relative to $\left[\mathrm{Ru}(\mathrm{bpy})_{3}\right]\left[\mathrm{PF}_{6}\right]_{2}$ and corrected for wavelength dependent detector sensitivity (Fig. S1). ${ }^{39,40}$ FT-IR spectra were recorded using a Perkin Elmer Spectrum Two FT-IR spectrometer equipped with an attenuated total reflectance (ATR) attachement.

\section{Cyclic Voltammetry}

Cyclic voltammetry experiments were performed with a Bioanalytical Systems Inc. (BASi) Epsilon potentiostat and analyzed using BASi Epsilon software. Typical electrochemical cells consisted of a three-electrode setup including a glassy carbon working electrode, platinum counter electrode, and silver pseudo reference electrode. Experiments were run at scan rates of $250 \mathrm{mV} \mathrm{s}^{-1}$ in degassed $\mathrm{CH}_{2} \mathrm{Cl}_{2}$ solutions of the analyte $(\sim 1 \mathrm{mM})$ and electrolyte $\left(0.1 \mathrm{M}\left[n \mathrm{Bu}_{4} \mathrm{~N}\right]\left[\mathrm{PF}_{6}\right]\right)$. Cyclic voltammograms were internally referenced against the ferrocene/ferrocenium redox couple $(\sim 1 \mathrm{mM}$ internal standard) and corrected for internal cell resistance using the BASi Epsilon software.

\section{Gel Permeation Chromatography (GPC)}

GPC experiments were conducted in chromatography-grade DMF at concentrations of $5 \mathrm{mg} \mathrm{mL}^{-1}$ using a Waters 2695 separations module equipped with a Waters 2414 differential refractometer and two PLgel $5 \mu \mathrm{m}$ mixed-D $(300 \mathrm{~mm} \times$ $7.5 \mathrm{~mm}$ ) columns from Polymer Laboratories connected in series. The calibration was performed using monodisperse polystyrene standards.

\section{Thermal Analysis}

Thermal degradation studies were performed using a TA Instruments Q50 TGA. The samples were placed in a platinum pan and heated at a rate of $10^{\circ} \mathrm{C} \mathrm{min}-1$ from $25^{\circ} \mathrm{C}$ to $1000{ }^{\circ} \mathrm{C}$

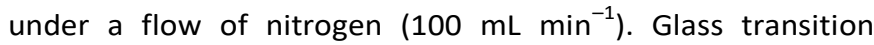
temperatures $\left(T_{g} s\right)$ were determined using Differential Scanning Calorimetry (DSC) on a TA Instruments DSC Q2000. The polymer samples were placed in an aluminum Tzero pan and heated to varying temperature ranges at $10{ }^{\circ} \mathrm{C} \mathrm{min}^{-1}$ under a flow of nitrogen $\left(50 \mathrm{~mL} \mathrm{~min}^{-1}\right)$ and cooled down to $-75^{\circ} \mathrm{C}$ at $10^{\circ} \mathrm{C} \mathrm{min}^{-1}$, before the sample underwent two more heating/cooling cycles. The glass transition temperatures were determined from the second heating/cooling cycle.

\section{Synthetic Procedures}

\section{Monomer BF2N}

PMDETA (0.005 g, $0.006 \mathrm{~mL}, 0.03 \mathrm{mmol}$ ) and azide-substituted norbornene $\mathbf{N}_{\mathbf{3}}-\mathbf{N}(0.162 \mathrm{~g}, 0.732 \mathrm{mmol}$ ) were dissolved in dry THF $(4 \mathrm{~mL})$ and the mixture was degassed via three freezepump-thaw cycles. Cul $(0.006 \mathrm{~g}, 0.03 \mathrm{mmol})$ was then added and the mixture was stirred for $15 \mathrm{~min}$ at $23^{\circ} \mathrm{C} . \mathrm{BF}_{2}$ complex HCC-BF2 $(0.214 \mathrm{~g}, 0.610 \mathrm{mmol})$ was then added and the reaction mixture was stirred at $23{ }^{\circ} \mathrm{C}$ for $2 \mathrm{~h}$. The solvent was removed in vacuo and the mixture purified by flash chromatography (silica gel); first toluene was used as eluent to remove purple and blue side products, then 1:1 toluene:EtOAc was added to the column and the purple product eluted. Removal of the solvent in vacuo afforded monomer BF2N as a dark-purple microcrystalline solid. Yield $=0.27 \mathrm{~g}, 77 \%$. M.p. 75-77 ${ }^{\circ} \mathrm{C} .{ }^{1} \mathrm{H}$ NMR $\left(399.8 \mathrm{MHz}, \mathrm{CDCl}_{3}\right): \delta$ 7.97-7.95 (m, 6H, aryl $\mathrm{CH}), 7.90($ br s, $1 \mathrm{H}$, triazole $\mathrm{CH}), 7.02-7.00(\mathrm{~m}, 2 \mathrm{H}$, aryl $\mathrm{CH})$, $6.23\left(\mathrm{dd}, 1 \mathrm{H},{ }^{3} J_{\mathrm{HH}}=6 \mathrm{~Hz},{ }^{3} J_{\mathrm{HH}}=3 \mathrm{~Hz},=\mathrm{CH}\right), 5.95\left(\mathrm{dd}, 1 \mathrm{H},{ }^{3} J_{\mathrm{HH}}=6\right.$ $\left.\mathrm{Hz},{ }^{3} \mathrm{~J}_{\mathrm{HH}}=3 \mathrm{~Hz},=\mathrm{CH}\right), 4.53\left(\mathrm{t},{ }^{3} \mathrm{~J}_{\mathrm{HH}}=7 \mathrm{~Hz}, 2 \mathrm{H}, \mathrm{CH}_{2}\right), 4.15-4.11$ $\left(\mathrm{m}, 2 \mathrm{H}, \mathrm{CH}_{2}\right), 3.92\left(\mathrm{~s}, 3 \mathrm{H}, \mathrm{OCH}_{3}\right), 3.22(\mathrm{br} \mathrm{s}, 1 \mathrm{H}, \mathrm{CH}), 2.99-2.94$ $(\mathrm{m}, 2 \mathrm{H}, \mathrm{CH}), 2.35-2.29\left(\mathrm{~m}, 2 \mathrm{H}, \mathrm{CH}_{2}\right), 1.96-1.90(\mathrm{~m}, 1 \mathrm{H}$, diastereotopic $\left.\mathrm{CH}_{2}\right), 1.48-1.41\left(\mathrm{~m}, 2 \mathrm{H}\right.$, diastereotopic $\left.\mathrm{CH}_{2}\right)$, 1.30-1.28 $\left(\mathrm{m}, 1 \mathrm{H}\right.$, diastereotopic $\left.\mathrm{CH}_{2}\right) .{ }^{13} \mathrm{C}\left\{{ }^{1} \mathrm{H}\right\} \quad \mathrm{NMR}$ $\left(150.7 \mathrm{MHz}, \mathrm{CDCl}_{3}\right): \delta 174.6,162.7,146.3,142.7,138.1,138.0$, $136.8,132.9,132.1,126.4,125.2,123.4,120.8,114.9,114.2$, $60.6,55.9,49.7,47.4,45.8,43.3,42.5,29.6,29.2 .{ }^{11} \mathrm{~B} N M R$ 
(128.3 MHz, $\left.\mathrm{CDCl}_{3}\right): \delta-0.7\left(\mathrm{t},{ }^{1} J_{\mathrm{BF}}=30 \mathrm{~Hz}\right) .{ }^{19} \mathrm{~F} \mathrm{NMR}$ (376.1 MHz, CDCl 3 ): $\delta-134.1$ (q, ${ }^{1} J_{\mathrm{FB}}=30 \mathrm{~Hz}$ ). FT-IR (ATR): 3138 (w), $2933(\mathrm{~m}), 2857(\mathrm{~m}), 2241(\mathrm{~m}), 1726(\mathrm{~s}), 1597(\mathrm{~s}), 1505(\mathrm{~m})$, $1334(\mathrm{~s}), 1261$ (s) $\mathrm{cm}^{-1}$. UV-vis $\left(\mathrm{CH}_{2} \mathrm{Cl}_{2}\right): \lambda_{\max } 561 \mathrm{~nm}(\varepsilon=$ $35,300 \mathrm{M}^{-1} \mathrm{~cm}^{-1}$ ). Mass Spec. (El, +ve mode): exact mass calculated for $\mathrm{C}_{28} \mathrm{H}_{27} \mathrm{BF}_{2} \mathrm{~N}_{8} \mathrm{O}_{3}$ : 572.2267; exact mass found: 572.2256; difference: $-1.9 \mathrm{ppm}$.

\section{Representative ROMP of DND}

Monomer DND (0.100 g, $0.476 \mathrm{mmol}$ ) was dissolved in $1.9 \mathrm{~mL}$ of dry and degassed (via three freeze-pump-thaw cycles) $\mathrm{N}, \mathrm{N}$ dimethylacetamide (DMA), and the solution was stirred at $23{ }^{\circ} \mathrm{C}$ for $15 \mathrm{~min}$. Meanwhile, G3 (0.013 g, $0.014 \mathrm{mmol}$ ) was dissolved in $0.3 \mathrm{~mL}$ of dry and degassed DMA. A $0.1 \mathrm{~mL}$ portion of the solution of $\mathbf{G} 3(0.004 \mathrm{~g}, 1 \mathrm{~mol} \%)$ was then added to the solution of monomer DND and stirred at $23^{\circ} \mathrm{C}$ for $6 \mathrm{~min}$. After $6 \mathrm{~min}$, ethyl vinyl ether $(0.857 \mathrm{~g}, 0.62 \mathrm{~mL}, 11.9 \mathrm{mmol})$ was added and the solution was stirred at $23{ }^{\circ} \mathrm{C}$ for $30 \mathrm{~min}$. The target polymer was purified by precipitation into pentane, isolated by centrifugation, and dried at $23{ }^{\circ} \mathrm{C}$ in vacuo for $16 \mathrm{~h}$ to afford PDND as a white solid. Yield $=0.075 \mathrm{~g}, 75 \%$. ${ }^{1} \mathrm{H}$ NMR (399.8 $\mathrm{MHz}_{\mathrm{CDCl}}$ ): $\delta 5.42$ and 5.24 (br m, 2H, =CH), 3.64 (br s, $\left.6 \mathrm{H}, \mathrm{OCH}_{3}\right), 3.38(\mathrm{br} \mathrm{s}, 1 \mathrm{H}, \mathrm{CH}), 2.98(\mathrm{br} \mathrm{m}, 1 \mathrm{H}, \mathrm{CH}), 2.85$ (br m, $2 \mathrm{H}, \mathrm{CH}$ ), 2.07 (br m, $1 \mathrm{H}$, diastereotopic $\mathrm{CH}_{2}$ ), 1.23 (br m, $1 \mathrm{H}$, diastereotopic $\mathrm{CH}_{2}$ ). GPC: $\mathrm{M}_{\mathrm{n}}=38,800 \mathrm{~g} \mathrm{~mol}^{-1}, \mathrm{M}_{\mathrm{w}}=$ 43,050 $\mathrm{g} \mathrm{mol}^{-1}, \ominus=1.11$.

\section{Representative ROMP of BF2N}

Monomer BF2N (0.050 g, $0.087 \mathrm{mmol}$ ) was dissolved in $1.9 \mathrm{~mL}$ of dry and degassed (via three freeze-pump-thaw cycles) DMA, and the solution was stirred at $23{ }^{\circ} \mathrm{C}$ for $15 \mathrm{~min}$. Meanwhile, G3 (0.003 g, 1 mol\%) was dissolved in $0.3 \mathrm{~mL}$ of dry and degassed DMA. A $0.1 \mathrm{~mL}$ portion of the solution of $\mathbf{G} 3(0.001 \mathrm{~g}$, $1 \mathrm{~mol} \%$ ) was then added to the solution of monomer BF2N and stirred at $23{ }^{\circ} \mathrm{C}$ for $1 \mathrm{~h}$. After $1 \mathrm{~h}$, ethyl vinyl ether $(0.157 \mathrm{~g}$, $2.18 \mathrm{mmol}$ ) was added and the solution was stirred at $23^{\circ} \mathrm{C}$ for $30 \mathrm{~min}$. The target polymer was purified by flash chromatography (THF, neutral alumina) before it was precipited into pentane, isolated by centrifugation, and dried at $23{ }^{\circ} \mathrm{C}$ in vacuo for $16 \mathrm{~h}$ to afford polymer PBF2N as a purple solid. Yield $=0.030 \mathrm{~g}, 66 \% .{ }^{1} \mathrm{H}$ NMR $\left(399.8 \mathrm{MHz}, \mathrm{CDCl}_{3}\right): \delta 7.97$ (br s, $1 \mathrm{H}$, triazole $\mathrm{CH}$ ), $7.84(\mathrm{br} \mathrm{s}, 6 \mathrm{H}$, aryl $\mathrm{CH}), 6.92(\mathrm{br} \mathrm{s}, 2 \mathrm{H}$, aryl $\mathrm{CH}), 5.35-5.25(2 \times \mathrm{br} \mathrm{m}, 2 \mathrm{H},=\mathrm{CH}), 4.45\left(\mathrm{br} \mathrm{s}, 2 \mathrm{H}, \mathrm{CH}_{2}\right)$, 4.07 (br s, $2 \mathrm{H}, \mathrm{CH}_{2}$ ), 3.84 (br s, $3 \mathrm{H}, \mathrm{OCH}_{3}$ ), 3.13 (br s, $1 \mathrm{H}, \mathrm{CH}$ ), 2.84 (br m, $2 \mathrm{H}, 2 \mathrm{CH}$ ), 2.24 (br s, $2 \mathrm{H}, \mathrm{CH}_{2}$ ), 1.90 (br m, $2 \mathrm{H}$, diastereotopic $\mathrm{CH}_{2}$ ), 1.71 (br m, $1 \mathrm{H}$, diastereotopic $\mathrm{CH}_{2}$ ), 1.30 (br $\mathrm{m}, 1 \mathrm{H}$, diastereotopic $\mathrm{CH}_{2}$ ). ${ }^{11} \mathrm{~B}$ NMR $\left(128.3 \mathrm{MHz}, \mathrm{CDCl}_{3}\right): \delta$ $-0.8\left(\mathrm{t},{ }^{1} J_{\mathrm{BF}}=30 \mathrm{~Hz}\right) .{ }^{19} \mathrm{~F} \mathrm{NMR}(376.1 \mathrm{MHz}, \mathrm{CDCl} 3): \delta-133.5(\mathrm{br}$ s). FT-IR (ATR): 3153 (w), 2952 (m), $2843(\mathrm{~m}), 2243(\mathrm{~m}), 1729$ (s), 1598 (s), $1506(\mathrm{~m}), 1343$ (s), 1263 (s) cm ${ }^{-1}$. UV-vis $\left(\mathrm{CH}_{2} \mathrm{Cl}_{2}\right)$ : $\lambda_{\max }=555 \mathrm{~nm} \quad\left(\varepsilon=27,500 \mathrm{M}^{-1} \mathrm{~cm}^{-1}\right)$. GPC: $\mathrm{M}_{\mathrm{n}}=$ $201,500 \mathrm{~g} \mathrm{~mol}^{-1}, \mathrm{M}_{\mathrm{w}}=264,300 \mathrm{~g} \mathrm{~mol}^{-1}, \oplus=1.31$.

Representative procedure for the preparation of random copolymers $(\text { PDND })_{m}-r-(\text { PBF2N })_{n}:(P D N D)_{m}-r-(P B F 2 N)_{n}\left(f_{B F 2 N}=0.50\right)$ Monomers BF2N (0.150 g, $0.262 \mathrm{mmol})$ and DND $(0.055 \mathrm{~g}$, $0.262 \mathrm{mmol}$ ) were dissolved in $3.9 \mathrm{~mL}$ of dry and degassed (via three freeze-pump-thaw cycles) DMA, and the solution was stirred at $23{ }^{\circ} \mathrm{C}$ for $15 \mathrm{~min}$. Meanwhile, G3 (0.009 g, $0.01 \mathrm{mmol}$ ) was dissolved in $0.4 \mathrm{~mL}$ of dry and degassed DMA. A $0.2 \mathrm{~mL}$ portion of the solution of $\mathbf{G} 3(0.005 \mathrm{~g}, 1 \mathrm{~mol} \%)$ was then added to the solution of monomers BF2N and DND and stirred at $23{ }^{\circ} \mathrm{C}$ for $12 \mathrm{~min}$. Ethyl vinyl ether (0.945 g, $0.690 \mathrm{~mL}, 13.1 \mathrm{mmol}$ ) was then added and the solution was stirred at $23{ }^{\circ} \mathrm{C}$ for $30 \mathrm{~min}$. The target polymer was purified by precipitation from pentane, isolated by centrifugation, and dried at $23{ }^{\circ} \mathrm{C}$ in vacuo for $16 \mathrm{~h}$ to afford (PDND) $)_{m}-r-(\text { PBF2N })_{n}$ $\left(f_{\mathrm{BF} 2 \mathrm{~N}}=0.50\right)$ as a purple solid. Yield $=0.248 \mathrm{~g}, 60 \% .{ }^{1} \mathrm{H} \mathrm{NMR}$ (399.8 MHz, $\left.\mathrm{CDCl}_{3}\right): \delta 7.90(\mathrm{br} \mathrm{m}, 7 \mathrm{H}$, triazole $\mathrm{CH}+$ aryl $\mathrm{CH})$, 6.97 (br s, $2 \mathrm{H}$, aryl $\mathrm{CH}), 5.41-5.23(2 \times \mathrm{br} \mathrm{m}, 4 \mathrm{H},=\mathrm{CH}), 4.49(\mathrm{br}$ $\left.\mathrm{s}, 2 \mathrm{H}, \mathrm{CH}_{2}\right), 4.11\left(\mathrm{br} \mathrm{s}, 2 \mathrm{H}, \mathrm{CH}_{2}\right), 3.88\left(\mathrm{br} \mathrm{s}, 3 \mathrm{H}, \mathrm{OCH}_{3}\right), 3.63(\mathrm{br}$ $\left.\mathrm{s}, 6 \mathrm{H}, \mathrm{OCH}_{3}\right), 3.35$ (br s, $\left.1 \mathrm{H}, \mathrm{CH}\right), 3.13(\mathrm{br} \mathrm{s}, 1 \mathrm{H}, \mathrm{CH}), 2.98-2.85$ (br m, 5H, CH), 2.29 (br s, 2H, CH $), 2.07-1.91$ (br m, 3H, diastereotopic $\mathrm{CH}_{2}$ ), 1.72 (br m, $1 \mathrm{H}$, diastereotopic $\mathrm{CH}_{2}$ ), 1.36$1.20\left(\mathrm{br} \mathrm{m}, 2 \mathrm{H}\right.$, diastereotopic $\left.\mathrm{CH}_{2}\right) .{ }^{11} \mathrm{~B} \mathrm{NMR}(128.4 \mathrm{MHz}$, $\left.\mathrm{CDCl}_{3}\right): \delta-0.7\left(\mathrm{t},{ }^{1} J_{\mathrm{BF}}=30 \mathrm{~Hz}\right) \cdot{ }^{19} \mathrm{~F} \mathrm{NMR}\left(376.4 \mathrm{MHz}, \mathrm{CDCl}_{3}\right): \delta$ -133.8 (br s). FT-IR (ATR): 2980 (w), 2951 (m), 2845 (m), 2240 $(\mathrm{m}), 1736(\mathrm{~s}), 1604(\mathrm{~s}), 1505(\mathrm{~m}), 1348(\mathrm{~s}), 1263(\mathrm{~s}) \mathrm{cm}^{-1}$. UV-vis $\left(\mathrm{CH}_{2} \mathrm{Cl}_{2}\right): \lambda_{\max }=558 \mathrm{~nm}$. GPC: $\mathrm{M}_{\mathrm{n}}=247,300 \mathrm{~g} \mathrm{~mol}^{-1}, \mathrm{M}_{\mathrm{w}}=$ $335,200 \mathrm{~g} \mathrm{~mol}^{-1}, \oplus=1.36$.

\section{$(\mathrm{PDND})_{\mathrm{m}}-r-(\mathrm{PBF} 2 \mathrm{~N})_{\mathrm{n}}\left(\mathrm{f}_{\mathrm{BF} 2 \mathrm{~N}}=\mathbf{0 . 1 5}\right)$}

From monomer BF2N (0.030 g, $0.053 \mathrm{mmol})$ and DND (0.056 g, $0.27 \mathrm{mmol})$. Yield $=0.810 \mathrm{~g}, 94 \% .{ }^{1} \mathrm{H} \mathrm{NMR}\left(399.8 \mathrm{MHz}, \mathrm{CDCl}_{3}\right)$ : $\delta 8.04$ (br s, $1 \mathrm{H}$, triazole $\mathrm{CH}), 7.95$ (br s, 6H, aryl $\mathrm{CH}), 7.00$ (br s, $2 \mathrm{H}$, aryl $\mathrm{CH}), 5.42-5.24(2 \times \mathrm{br} \mathrm{m}, 13 \mathrm{H},=\mathrm{CH}), 4.51(\mathrm{br} \mathrm{s}, 2 \mathrm{H}$, $\left.\mathrm{CH}_{2}\right), 4.14\left(\mathrm{br} \mathrm{s}, 2 \mathrm{H}, \mathrm{CH}_{2}\right), 3.91\left(\mathrm{br} \mathrm{s}, 3 \mathrm{H}, \mathrm{OCH}_{3}\right), 3.63(\mathrm{br} \mathrm{s}, 33 \mathrm{H}$, $\mathrm{OCH}_{3}$ ), 3.38 (br s, 6H, CH), 3.13 (br s, $\left.1 \mathrm{H}, \mathrm{CH}\right), 2.98-2.85$ (br m, $19 \mathrm{H}, \mathrm{CH}$ ), 2.29 (br s, $2 \mathrm{H}, \mathrm{CH}_{2}$ ), 2.06 (br m, 6H, diastereotopic $\left.\mathrm{CH}_{2}\right), 1.90\left(\mathrm{br} \mathrm{m}, 2 \mathrm{H}\right.$, diastereotopic $\left.\mathrm{CH}_{2}\right), 1.72(\mathrm{br} \mathrm{m}, 1 \mathrm{H}$, diastereotopic $\mathrm{CH}_{2}$ ), 1.38 (br m, $1 \mathrm{H}$, diastereotopic $\left.\mathrm{CH}_{2}\right), 1.30-$ 1.16 (br m, 5.5H, diastereotopic $\mathrm{CH}_{2}$ ). ${ }^{11} \mathrm{~B} \mathrm{NMR}(128.3 \mathrm{MHz}$, $\left.\mathrm{CDCl}_{3}\right): \delta-0.7\left(\mathrm{t},{ }^{1} \mathrm{~J}_{\mathrm{BF}}=31 \mathrm{~Hz}\right) .{ }^{19} \mathrm{~F} \mathrm{NMR}\left(376.1 \mathrm{MHz}, \mathrm{CDCl}_{3}\right): \delta-$ 134.0 (br s). FT-IR (ATR): 3002 (w), 2951 (m), 2852 (w), 1743 (s), 1597 (s), 1439 (m), 1344 (s), 1267 (s) cm ${ }^{-1}$. UV-vis $\left(\mathrm{CH}_{2} \mathrm{Cl}_{2}\right)$ : $\lambda_{\max }=560 \mathrm{~nm}$. GPC: $M_{n}=90,900 \mathrm{~g} \mathrm{~mol}^{-1}, M_{w}=$ $107,000 \mathrm{~g} \mathrm{~mol}^{-1}, \oplus=1.18$.

\section{$(\mathrm{PDND})_{\mathrm{m}}-r-(\mathrm{PBF} 2 \mathrm{~N})_{\mathrm{n}}\left(\mathrm{f}_{\mathrm{BF} 2 \mathrm{~N}}=\mathbf{0 . 0 8}\right)$}

From monomer BF2N $(0.021 \mathrm{~g}, 0.036 \mathrm{mmol})$ and DND (0.076 g, $0.36 \mathrm{mmol})$. Yield $=0.85 \mathrm{~g}, 88 \% .{ }^{1} \mathrm{H} \mathrm{NMR}\left(399.8 \mathrm{MHz}, \mathrm{CDCl}_{3}\right): \delta$ 8.05 (br s, $1 \mathrm{H}$, triazole $\mathrm{CH}$ ), 7.94 (br s, 6H, aryl $\mathrm{CH}), 7.01$ (br m, $2 \mathrm{H}$, aryl $\mathrm{CH}), 5.42-5.24(2 \times \mathrm{br} \mathrm{m}, 26.6 \mathrm{H},=\mathrm{CH}), 4.51(\mathrm{br} \mathrm{s}, 2 \mathrm{H}$, $\mathrm{CH}_{2}$ ), 4.14 (br s, 2H, $\mathrm{CH}_{2}$ ), 3.91 (br s, $\left.3 \mathrm{H}, \mathrm{OCH}_{3}\right), 3.63$ (br s, 74H, $\left.\mathrm{OCH}_{3}\right), 3.37$ (br s, $\left.12 \mathrm{H}, \mathrm{CH}\right), 3.14(\mathrm{br} \mathrm{s}, 1 \mathrm{H}, \mathrm{CH}), 3.02-2.84(\mathrm{br}$ $\mathrm{m}, 36 \mathrm{H}, \mathrm{CH}), 2.28\left(\mathrm{br} \mathrm{s}, 2 \mathrm{H}, \mathrm{CH}_{2}\right), 2.07(\mathrm{br} \mathrm{m}, 12 \mathrm{H}$, diastereotopic $\mathrm{CH}_{2}$ ), 1.90 (br m, 2H, diastereotopic $\left.\mathrm{CH}_{2}\right), 1.73$ (br $\mathrm{m}, 1 \mathrm{H}$, diastereotopic $\mathrm{CH}_{2}$ ), 1.33-1.19 (br $\mathrm{m}, 13 \mathrm{H}$, diastereotopic $\left.\mathrm{CH}_{2}\right) .{ }^{11} \mathrm{~B}$ NMR $\left(128.3 \mathrm{MHz}, \mathrm{CDCl}_{3}\right): \delta-0.7\left(\mathrm{t},{ }^{1} \mathrm{~J}_{\mathrm{BF}}\right.$ $=33 \mathrm{~Hz}) \cdot{ }^{19} \mathrm{~F} \mathrm{NMR}\left(376.1 \mathrm{MHz}, \mathrm{CDCl}_{3}\right): \delta-134.0(\mathrm{br} \mathrm{s})$. FT-IR (ATR): $2998(\mathrm{~m}), 2952(\mathrm{~m}), 2850(\mathrm{~m}), 1733$ (s), $1599(\mathrm{w}), 1436$ (m), $1363(\mathrm{w}), 1264(\mathrm{~m}) \mathrm{cm}^{-1}$. UV-vis $\left(\mathrm{CH}_{2} \mathrm{Cl}_{2}\right): \lambda_{\max }=561 \mathrm{~nm}$. GPC: $M_{n}=77,500 \mathrm{~g} \mathrm{~mol}^{-1}, M_{w}=88,800 \mathrm{~g} \mathrm{~mol}^{-1}, \oslash=1.15$. 
Representative procedure for the preparation of block copolymers $(\text { PDND })_{\mathrm{m}}-b-(\text { PBF2N })_{n}$ : (PDND) $)_{m}-b-(\text { PBF2N })_{n}\left(f_{\text {BF2N }}=0.48\right)$

Monomer DND (0.150 g, $0.713 \mathrm{mmol})$ was dissolved in $2.9 \mathrm{~mL}$ of dry and degassed (via three freeze-pump-thaw cycles) DMA, and the solution was stirred at $23{ }^{\circ} \mathrm{C}$ for $15 \mathrm{~min}$. Meanwhile, G3 $(0.019 \mathrm{~g}, 0.021 \mathrm{mmol}$ ) was dissolved in $0.3 \mathrm{~mL}$ of dry and degassed DMA, and BF2N (0.272 $\mathrm{g}, 0.475 \mathrm{mmol})$ was dissolved in $0.64 \mathrm{~mL}$ of dry and degassed DMA. A $0.1 \mathrm{~mL}$ portion of the solution of $\mathbf{G} 3(0.0063 \mathrm{~g}, 1 \mathrm{~mol} \%)$ was then added to the solution of monomer DND and stirred at $23{ }^{\circ} \mathrm{C}$ for $6 \mathrm{~min}$. After $6 \mathrm{~min}, 1 \mathrm{~mL}$ of the reaction mixture was removed and added to ethyl vinyl ether $(0.429 \mathrm{~g}, 0.31 \mathrm{~mL}, 5.95 \mathrm{mmol})$ and stirred at $23{ }^{\circ} \mathrm{C}$ for $30 \mathrm{~min}$ before PDND was precipitated from pentane, isolated by centrifugation, and dried in vacuo for $16 \mathrm{~h}$ to afford polymer PDND as a white solid. GPC: $M_{n}=38,800 \mathrm{~g} \mathrm{~mol}^{-1}, M_{w}$ $=43,050 \mathrm{~g} \mathrm{~mol}^{-1}, Ð=1.11$. After removal of the $1 \mathrm{~mL}$ aliquot of the reactant mixture, the BF2N solution was added and stirred at $23{ }^{\circ} \mathrm{C}$ for $12 \mathrm{~min}$. Ethyl vinyl ether $(0.857 \mathrm{~g}, 0.62 \mathrm{~mL}$, $11.9 \mathrm{mmol}$ ) was then added and the solution was stirred at $23{ }^{\circ} \mathrm{C}$ for $30 \mathrm{~min}$. The target polymer was purified by precipitation into pentane, isolated by centrifugation, and dried at $23^{\circ} \mathrm{C}$ in vacuo for $16 \mathrm{~h}$ to afford polymer (PDND) $\mathrm{m}^{-b}$ $(\text { PBF2N })_{\mathbf{n}}\left(f_{\mathrm{BF} 2 \mathrm{~N}}=0.48\right)$ as a purple solid in quantitative yield. ${ }^{1} \mathrm{H}$ NMR (399.8 MHz, $\mathrm{CDCl}_{3}$ ): $\delta 7.97$ (br s, $1 \mathrm{H}$, triazole $\mathrm{CH}$ ), 7.83 (br $\mathrm{m}, 6 \mathrm{H}$, aryl $\mathrm{CH}), 6.91(\mathrm{br} \mathrm{s}, 2 \mathrm{H}$, aryl $\mathrm{CH}), 5.42-5.24(2 \times \mathrm{br} \mathrm{m}$, $4.2 \mathrm{H},=\mathrm{CH}$ ), $4.44\left(\mathrm{br} \mathrm{s}, 2 \mathrm{H}, \mathrm{CH}_{2}\right.$ ), 4.07 (br s, $2 \mathrm{H}, \mathrm{CH}_{2}$ ), 3.83 (br s, $\left.3 \mathrm{H}, \mathrm{OCH}_{3}\right), 3.64\left(\mathrm{br} \mathrm{s}, 6.6 \mathrm{H}, \mathrm{OCH}_{3}\right), 3.38(\mathrm{br} \mathrm{s}, 1 \mathrm{H}, \mathrm{CH}), 3.13(\mathrm{br}$ $\mathrm{s}, 1 \mathrm{H}, \mathrm{CH}), 2.98-2.85(\mathrm{br} \mathrm{m}, 5 \mathrm{H}, \mathrm{CH}), 2.25$ (br s, $2 \mathrm{H}, \mathrm{CH}_{2}$ ), 2.05 (br $\mathrm{m}, 1 \mathrm{H}$, diastereotopic $\mathrm{CH}_{2}$ ), 1.92 (br $\mathrm{m}, 2 \mathrm{H}$, diastereotopic $\left.\mathrm{CH}_{2}\right), 1.72\left(\mathrm{br} \mathrm{m}, 1 \mathrm{H}\right.$, diastereotopic $\left.\mathrm{CH}_{2}\right), 1.34-1.16(\mathrm{br} \mathrm{m}, 2 \mathrm{H}$, diastereotopic $\left.\mathrm{CH}_{2}\right) .{ }^{11} \mathrm{~B} \mathrm{NMR}\left(128.3 \mathrm{MHz}, \mathrm{CDCl}_{3}\right): \delta-0.8\left(\mathrm{t},{ }^{1} J_{\mathrm{BF}}\right.$ $=30 \mathrm{~Hz}) \cdot{ }^{19} \mathrm{~F} \mathrm{NMR}\left(376.1 \mathrm{MHz}, \mathrm{CDCl}_{3}\right): \delta-133.4$ (br s). ). FT-IR (ATR): $3138(\mathrm{w}), 2955$ (m), $2849(\mathrm{~m}), 2241(\mathrm{~m}), 1733$ (s), 1597 (s), $1505(\mathrm{~m}), 1343(\mathrm{~s}), 1261(\mathrm{~s}) \mathrm{cm}^{-1}$. UV-vis $\left(\mathrm{CH}_{2} \mathrm{Cl}_{2}\right): \lambda_{\max }=$ $559 \mathrm{~nm}$. GPC: $\mathrm{M}_{\mathrm{n}}=204,300 \mathrm{~g} \mathrm{~mol}^{-1}, \mathrm{M}_{\mathrm{w}}=295,400 \mathrm{~g} \mathrm{~mol}^{-1}, \Theta=$ 1.45 .

\section{$(\text { PDND })_{m}-b-(\text { PBF2N })_{n}\left(f_{\mathrm{BF} 2 \mathrm{~N}}=\mathbf{0 . 1 3}\right)$}

From monomer DND (0.250 g, $1.19 \mathrm{mmol})$ and BF2N (0.109 g, $0.190 \mathrm{mmol}$ ). The aliquot removed at $6 \mathrm{~min}$ yielded polymer PDND. GPC: $M_{n}=54,910 \mathrm{~g} \mathrm{~mol}^{-1}, M_{w}=62,700 \mathrm{~g} \mathrm{~mol}^{-1}, \oplus=$ 1.14. The final reaction mixture afforded polymer $(P D N D)_{m}-b-$ $(\text { PBF2N })_{n}\left(f_{B F 2 N}=0.13\right)$ in quantitative yield. ${ }^{1} \mathrm{H}$ NMR $(399.8$ $\mathrm{MHz} \mathrm{CDCl}_{3}$ ): $\delta 7.97$ (br s, $1 \mathrm{H}$, triazole $\left.\mathrm{CH}\right), 7.85$ (br s, 6H, aryl $\mathrm{CH}), 6.93(\mathrm{br} \mathrm{s}, 2 \mathrm{H}$, aryl $\mathrm{CH}), 5.43-5.24(2 \times \mathrm{br} \mathrm{m}, 15.8 \mathrm{H},=\mathrm{CH})$, 4.46 (br s, $\left.2 \mathrm{H}, \mathrm{CH}_{2}\right), 4.08$ (br s, $\left.2 \mathrm{H}, \mathrm{CH}_{2}\right), 3.85\left(\mathrm{br} \mathrm{s}, 3 \mathrm{H}, \mathrm{OCH}_{3}\right)$, $3.64\left(\mathrm{br} \mathrm{s}, 41 \mathrm{H}, \mathrm{OCH}_{3}\right), 3.38(\mathrm{br} \mathrm{s}, 7 \mathrm{H}, \mathrm{CH}), 3.13(\mathrm{br} \mathrm{s}, 1 \mathrm{H}, \mathrm{CH})$ 3.02-2.85 (br m, 21H, CH), 2.25 (br s, 2H, $\mathrm{CH}_{2}$ ), 2.07 (br m, 7H, diastereotopic $\left.\mathrm{CH}_{2}\right), 1.91$ (br m, $2 \mathrm{H}$, diastereotopic $\left.\mathrm{CH}_{2}\right), 1.70$ (br $\mathrm{m}, 1 \mathrm{H}$, diastereotopic $\mathrm{CH}_{2}$ ), 1.33-1.14 (br $\mathrm{m}, 8 \mathrm{H}$, diastereotopic $\left.\mathrm{CH}_{2}\right) \cdot{ }^{11} \mathrm{~B} \mathrm{NMR}\left(128.3 \mathrm{MHz}, \mathrm{CDCl}_{3}\right): \delta-0.8\left(\mathrm{t},{ }^{1} J_{\mathrm{BF}}\right.$ $=30 \mathrm{~Hz}$ ). ${ }^{19} \mathrm{~F} \mathrm{NMR}\left(376.1 \mathrm{MHz}, \mathrm{CDCl}_{3}\right): \delta-133.5$ (br s). FT-IR (ATR): 2989 (w), 2950 (m), 2849 (w), 2240 (s), 1733 (s), 1599 (s), $1436(\mathrm{~m}), 1345(\mathrm{~s}), 1263(\mathrm{~s}) \mathrm{cm}^{-1}$. UV-vis $\left(\mathrm{CH}_{2} \mathrm{Cl}_{2}\right): \lambda_{\max }=$ $559 \mathrm{~nm}$. GPC: $\mathrm{M}_{\mathrm{n}}=104,000 \mathrm{~g} \mathrm{~mol}^{-1}, \mathrm{M}_{\mathrm{w}}=126,300 \mathrm{~g} \mathrm{~mol}^{-1}, \oslash=$ 1.21 .
$(\mathrm{PDND})_{\mathrm{m}}-b-(\mathrm{PBF} 2 \mathrm{~N})_{\mathrm{n}}\left(\mathrm{f}_{\mathrm{BF} 2 \mathrm{~N}}=\mathbf{0 . 0 7}\right)$

From monomer DND (0.300 g, $1.43 \mathrm{mmol})$ and BF2N (0.068 g, $0.12 \mathrm{mmol})$. The aliquot removed at $6 \mathrm{~min}$ yielded polymer PDND. GPC: $M_{n}=42,800 \mathrm{~g} \mathrm{~mol}^{-1}, M_{w}=48,600 \mathrm{~g} \mathrm{~mol}^{-1}, \emptyset=$ 1.13. The second solution afforded polymer (PDND) $\mathrm{m}^{-\boldsymbol{b}} \boldsymbol{b}$ $(\text { PBF2N })_{n}\left(f_{B F 2 N}=0.07\right)$ in quantitative yield. ${ }^{1} \mathrm{H}$ NMR $(399.8$ $\mathrm{MHz}, \mathrm{CDCl}_{3}$ ): $\delta 7.98(\mathrm{br} \mathrm{s}, 1 \mathrm{H}$, triazole $\mathrm{CH}), 7.89(\mathrm{br} \mathrm{s}, 6 \mathrm{H}$, aryl $\mathrm{CH}), 6.96(\mathrm{br} \mathrm{s}, 2 \mathrm{H}$, aryl $\mathrm{CH}), 5.43-5.24(2 \times \mathrm{br} \mathrm{m}, 27 \mathrm{H},=\mathrm{CH})$, 4.47 (br s, $2 \mathrm{H}, \mathrm{CH}_{2}$ ), 4.09 (br s, $2 \mathrm{H}, \mathrm{CH}_{2}$ ), 3.88 (br s, $3 \mathrm{H}, \mathrm{OCH}_{3}$ ), 3.64 (br s, 73H, OCH $\mathrm{OCH}_{3}, 3.38$ (br s, 13H, $\left.\mathrm{CH}\right), 3.13(\mathrm{br} \mathrm{s}, 1 \mathrm{H}, \mathrm{CH})$, 3.02-2.85 (br m, 4OH, CH), 2.27 (br s, $2 \mathrm{H}, \mathrm{CH}_{2}$ ), 2.07 (br m, $13 \mathrm{H}$, diastereotopic $\left.\mathrm{CH}_{2}\right), 1.91\left(\mathrm{br} \mathrm{m}, 2 \mathrm{H}\right.$, diastereotopic $\mathrm{CH}_{2}$ ), $1.70\left(\mathrm{br} \mathrm{m}, 1 \mathrm{H}\right.$, diastereotopic $\left.\mathrm{CH}_{2}\right), 1.30-1.17(\mathrm{br} \mathrm{m}, 14 \mathrm{H}$, diastereotopic $\left.\mathrm{CH}_{2}\right) .{ }^{11} \mathrm{~B} N M R\left(128.3 \mathrm{MHz}, \mathrm{CDCl}_{3}\right): \delta-0.7\left(\mathrm{t},{ }^{1} J_{\mathrm{BF}}\right.$ $=30 \mathrm{~Hz}) \cdot{ }^{19} \mathrm{~F}$ NMR $\left(376.1 \mathrm{MHz}, \mathrm{CDCl}_{3}\right): \delta-134.0$ (br s). FT-IR (ATR): 3000 (w), $2951(\mathrm{~m}), 2848(\mathrm{~m}), 1733$ (s), $1599(\mathrm{~m}), 1436$ (s), $1362(\mathrm{~m}), 1264(\mathrm{~s}) \mathrm{cm}^{-1}$. UV-vis $\left(\mathrm{CH}_{2} \mathrm{Cl}_{2}\right): \lambda_{\max }=559 \mathrm{~nm}$. GPC: $\mathrm{M}_{\mathrm{n}}=66,050 \mathrm{~g} \mathrm{~mol}^{-1}, \mathrm{M}_{\mathrm{w}}=76,400 \mathrm{~g} \mathrm{~mol}^{-1}, \oplus=1.16$.

\section{Synthesis and Molecular Weight Determination}

The monomers chosen for this study, due to their synthetic accessibility, were cis-dimethyl-5-norbornene-exo-2,3dicarboxylate $^{37}$ (DND) and the endo-isomer of an esterfunctionalized norbornene bearing an asymmetric $\mathrm{BF}_{2} 3-$ cyanoformazanate complex (BF2N, Fig. S2, S3). The latter was specifically targeted due to the generally higher intensity emission exhibited by $\mathrm{BF}_{2}$ complexes of 3-cyanoformazanate ligands, and was prepared via a copper-assisted alkyne-azide cycloaddition reaction between an azide-functionalized norbornene $^{35}\left(\mathbf{N}_{\mathbf{3}}-\mathbf{N}\right)$ and an alkyne-functionalized $\mathrm{BF}_{2}$ formazanate complex ${ }^{36}$ (HCC-BF2) according to Scheme 1.

Homopolymers PDND and PBF2N and random copolymers $(\text { PDND })_{m}-r-(\text { PBF2N })_{n}$ were prepared by ROMP in DMA at $23^{\circ} \mathrm{C}$

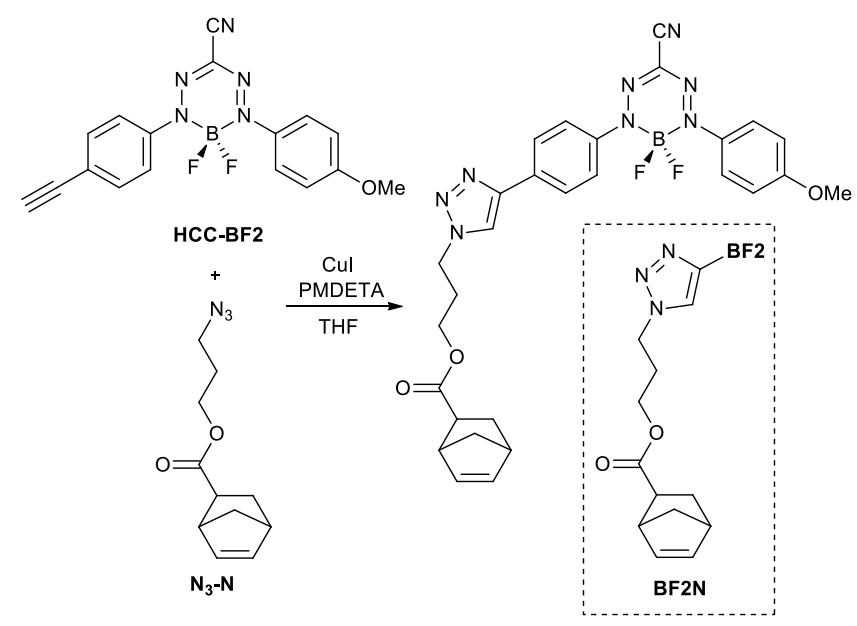

Scheme 1 Synthesis of monomer BF2N. The space-saving representation of BF2N is shown inside the dashed box. 


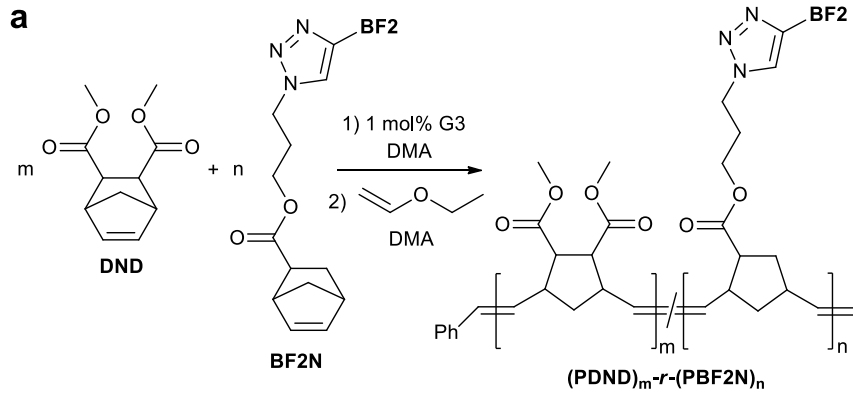

b

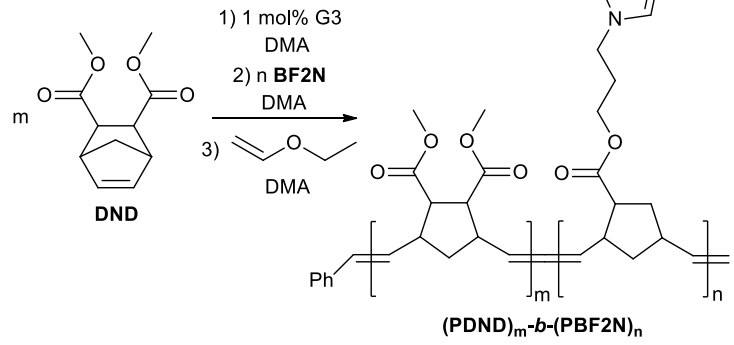

Scheme 2 Synthesis of (a) homopolymers PDND $(n=0), \operatorname{PBF2N}(m=0)$, and random copolymers (PDND) $)_{m}-r-(\text { PBF2N })_{n}$ and (b) block copolymers (PDND) $)_{m}-b-$ $(\text { PBF2N })_{n}$.

with a total monomer:G3 catalyst ratio of 100:1 (Scheme 2a, Fig. S4-S10). Hompolymerization reactions were monitored using GPC in DMF relative to monodisperse polystyrene standards in order to establish the time required to convert the respective monomers to homopolymers (Fig. S11). Lightscattering methods could not be employed due to absorption of the excitation laser employed $(631 \mathrm{~nm})$. The data collected were consistent with the fact that limited side and/or coupling reactions were occurring in solution and revealed a plateau in molecular weight after approximately $3 \mathrm{~min}$ for PDND and 10 min for PBF2N. These results were in agreement with the findings of previous studies of the ROMP of closely related monomers comprised of $\mathrm{BF}_{2}$ triarylformazanate complexes. ${ }^{29}$ In the case of the random and block copolymers, 1:1, 5:1, and 10:1 ratios of DND to BF2N were employed. Block copolymers were prepared under similar conditions by first combining DND with $G 3$ in a 100:1 ratio and then adding the desired amount of BF2N to produce (PDND) ${ }_{m}-\boldsymbol{b}-($ PBF2N) $n$ (Scheme $2 b$, Fig. S12-S14).

Upon inspection of the GPC data collected for the polymers described above (Table 1, Fig. S11 and S15), it became clear that the molecular weights of polymers containing BF2N repeating units were severely overestimated. We based this conclusion on the fact that the molecular weight distributions remain relatively narrow as the reaction progressed $(\theta=1.18$ 1.31) with little or no sign of high- and/or low-molecular weight shoulders, that may have arisen from termination reactions, in the respective GPC traces. Furthermore, for comparison, GPC data were collected for monomer BF2N, revealing a $M_{n}$ of $4850 \mathrm{~g} \mathrm{~mol}^{-1}$, roughly $8.5 \times$ that of the calculated molecular weight of $572.37 \mathrm{~g} \mathrm{~mol}^{-1}$. Thus, while these data have some utility in providing relative comparisons
Table 1 Molecular Weight Data for PDND, PBF2N, (PDND) $)_{m}-r-(\text { PBF2N })_{n}$, and (PDND) $)_{m}-b-($ PBF2N)

\begin{tabular}{ccccc}
\hline Polymer & $\mathrm{f}_{\mathrm{BF} 2 \mathrm{~N}}{ }^{a}$ & $\mathrm{M}_{\mathrm{n}}\left(\mathrm{g} \mathrm{mol}^{-1}\right)^{b}$ & $\mathrm{M}_{\mathrm{w}}\left(\mathrm{g} \mathrm{mol}^{-1}\right)^{b}$ & $\theta^{b}$ \\
\hline PDND & 0 & 38,800 & 43,050 & 1.11 \\
PBF2N & 1 & 201,500 & 264,300 & 1.31 \\
(PDND) $_{\mathrm{m}-\boldsymbol{r} \text {-(PBF2N) }}$ & 0.50 & 247,300 & 335,200 & 1.36 \\
& 0.15 & 90,900 & 107,000 & 1.18 \\
& 0.08 & 77,500 & 88,800 & 1.15 \\
(PDND) $_{\mathrm{m}-\boldsymbol{b} \text {-(PBF2N) }}$ & 0.48 & 204,300 & 295,400 & 1.45 \\
& 0.13 & 104,000 & 126,300 & 1.21 \\
& 0.07 & 66,050 & 76,400 & 1.16 \\
\hline
\end{tabular}

${ }^{a}$ Determined by relative integrations of isolated ${ }^{1} \mathrm{H}$ NMR spectroscopic signals. ${ }^{b}$ Determined by GPC analysis (vs. polystyrene standards) in DMF.

between the various polymers and assessing the breadth of the molecular weight distributions, they provide little accurate information about the total number of repeating units and no information about the relative ratio of DND and BF2N repeating units present in the copolymers. In the context of this work, the relative ratios of the DND and BF2N repeating units in the various polymers produced is far more informative. In order to reliably determine the molar ratio of repeating units, we turned to ${ }^{1} \mathrm{H}$ NMR integration data. Specifically, we compared the integration of the broad, isolated singlet centred at $c a$. $6.9 \mathrm{ppm}$ corresponding to 2 aryl protons from the $\mathrm{BF}_{2}$ formazanate complex and the total integration of the broad signals detected between 5.5 and 5.0 ppm, which correspond to the alkene protons arising from both repeating units in the polymer backbones (Table 1 ). These data, expressed as the mole fraction of BF2N repeating units $\left(f_{B F 2 N}\right)$, will be used throughout the remainder of this manuscript to identify the specific polymers being discussed.

\section{Thermal Analysis}

Thermal gravimetric analysis (TGA) of homopolymers PBF2N and PDND along with the corresponding random and block copolymers was used to assess their thermal stability from 25$1000{ }^{\circ} \mathrm{C}$ (Fig. S16). PBF2N did not lose significant mass until temperatures of $c a .225{ }^{\circ} \mathrm{C}$ were reached, while PDND was thermally stable up to a temperature of $c a .360^{\circ} \mathrm{C}$. At $1000^{\circ} \mathrm{C}$, $36 \%$ and $12 \%$ of the overall mass was retained for PBF2N and PDND, respectively. TGA data for the corresponding random and block copolymers exhibited features intermediate to those described above. Generally, thermal stability increased as $f_{B F 2 N}$ decreased.

Differential scanning calorimetry (DSC) studies of PDND revealed a $\mathrm{T}_{\mathrm{g}}$ of $83^{\circ} \mathrm{C}$ while PBF2N had a $\mathrm{T}_{\mathrm{g}}$ of $136^{\circ} \mathrm{C}$ (Fig. 1). The DSC thermograms of random copolymers (PDND) $\mathrm{m}^{-r}$ (PBF2N) $n$ were comprised of a single glass transition, consistent with their proposed structures, with $\mathrm{T}_{\mathrm{g}} \mathrm{s}$ increasing as $f_{B F 2 N}$ increased (Fig. 1a). Similar data were collected for block copolymers (PDND) $)_{m}-\boldsymbol{b}-(\text { PBF2N })_{n}$, and aside from the block polymer with $f_{\mathrm{BF} 2 \mathrm{~N}}=0.07$ where the BF2N content was too low to observe a corresponding $T_{g}$, the thermograms were 
a

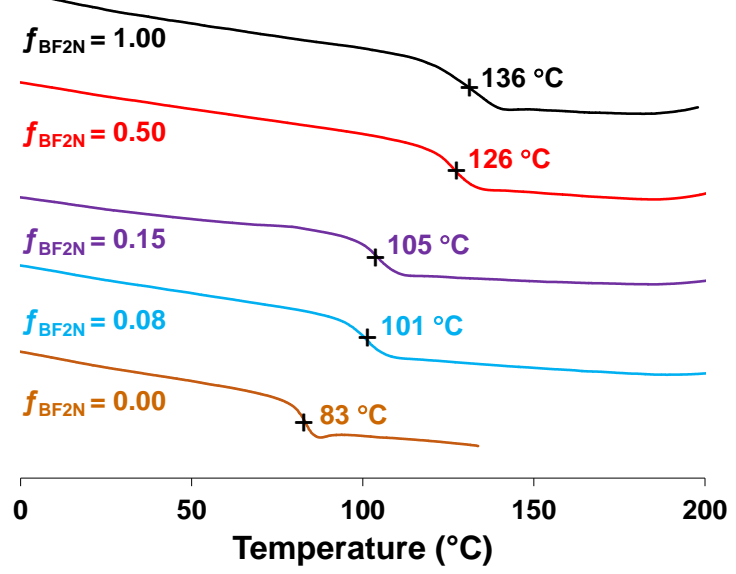

b

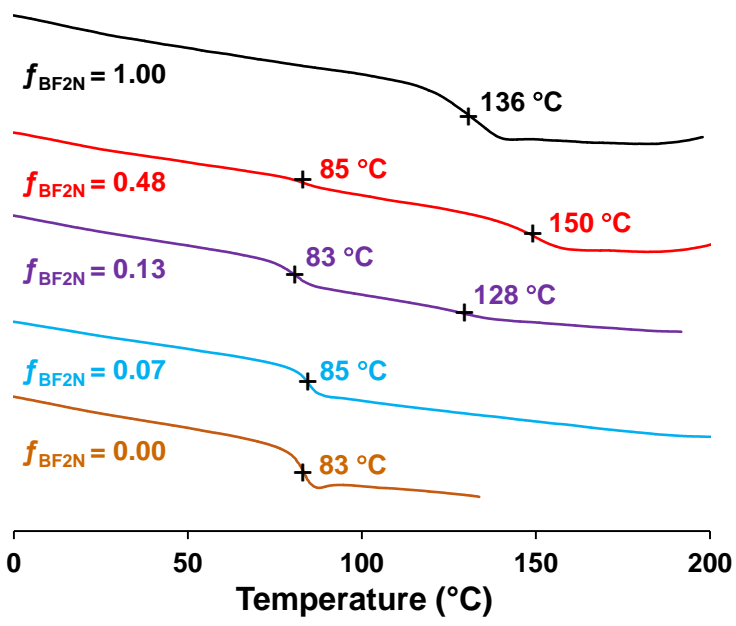

Fig. 1 DSC thermograms collected for homopolymers PBF2N and DND, (a) random copolymers $(\text { PDND })_{m}-r-(\text { PBF2N })_{n}$, and (b) block copolymers $(\text { PDND })_{m}-b-(\text { PBF2N) })_{n}$.

comprised of two $\mathrm{T}_{\mathrm{g}} \mathrm{s}$, as expected for the block architectures (Fig. 1b). No melt or crystallization events were observed for any of the polymers described in this study and $T_{g}$ values were determined from first derivative plots.

\section{Absorption/Emission Spectroscopy and Cyclic Voltammetry}

The UV-vis absorption spectra collected for monomer BF2N, homopolymer PBF2N, and random copolymers (PDND) $)_{m}^{-r}$ (PBF2N) $)_{n}$ are shown in Fig. 2 and the data summarized in Table 2. Each of the polymers produced in this study absorb strongly

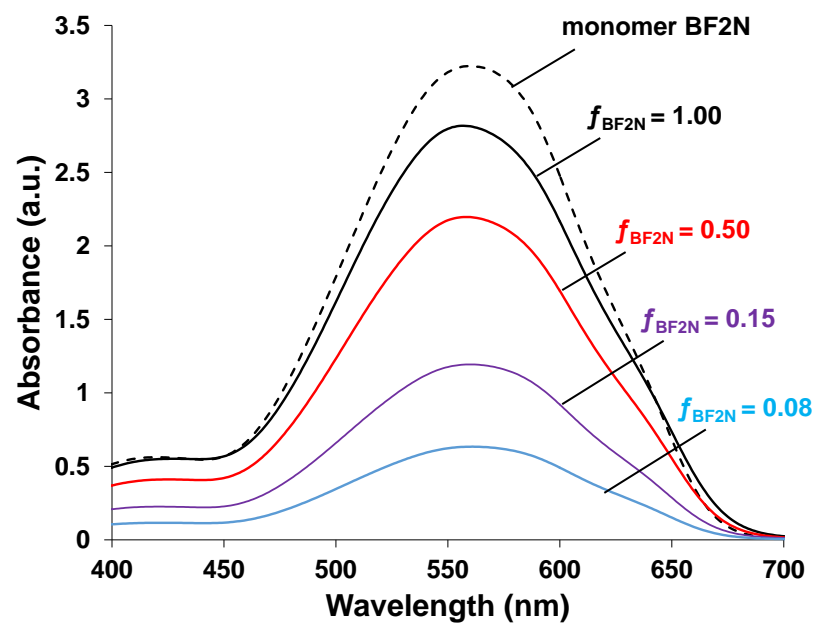

Fig. 2 UV-vis absorption spectra of monomer BF2N, homopolymer PBF2N, and random copolymers (PDND) $)^{-r}-(\text { PBF2N) })_{n}$ recorded for $0.05 \mathrm{mg} \mathrm{mL}^{-1} \mathrm{CH}_{2} \mathrm{Cl}_{2}$ solutions.

at $\lambda_{\max }$ of approximately $560 \mathrm{~nm}$ in $\mathrm{CH}_{2} \mathrm{Cl}_{2}$, as observed for related molecular species. ${ }^{32,36}$ The intensity of these absorption maxima did not increase linearly with $f_{B F 2 N}$ in the spectra collected for random copolymers (PDND) $m^{-r}-(\text { PBF2N) })_{n}$. We attribute this trend to the randomly coiled polymer chains in solution creating localized regions of high chromophore (i.e., BF2N repeating unit) concentration. In these regions, a single chromophore may act as a mask by absorbing incident radiation before it is able to reach other chromophores in close proximity. This effect appears to become more pronounced as $f_{B F 2 N}$ increases, and is most dramatic when comparing the absorbance spectra of monomer BF2N and homopolymer PBF2N. A similar trend was observed for block copolymers (PDND) $m_{m}-\boldsymbol{b}-(\text { PBF2N) })_{n}$ (Fig. S17). Based on the qualitatively similar shapes of the absorption spectra and the relatively constant $\lambda_{\max }$ values observed, we conclude that no significant degree of $\pi$ stacking is present in the solutions analysed.

The emission spectra collected for monomer BF2N, homopolymer PBF2N, random copolymers (PDND) $m^{-r}$ $(\text { PBF2N })_{n}$, and block copolymers (PDND) $)_{m}-b-(\text { PBF2N })_{n}$ revealed a significantly different trend (Fig. 3, Table 2). Each of the polymers studied yielded an emission band centred at $c a$. $665 \mathrm{~nm}$ and exhibited Stokes shifts $\left(\mathrm{U}_{\mathrm{ST}}\right.$ ) between 102-108 nm (2742-2897 $\left.\mathrm{cm}^{-1}\right)$, consistent with similar molecular species. ${ }^{32,36}$ The estimated quantum yields of fluorescence $\left(\Phi_{\mathrm{F}}\right)$

Table 2 Spectroscopic and electrochemical data obtained for monomer BF2N, hompolymer PBF2N, random copolymers (PDND) ${ }_{m}-r$-(PBF2N) ${ }_{n}$, and block copolymers (PDND) $)_{m}-b-($ PBF2N)

\begin{tabular}{|c|c|c|c|c|c|c|c|c|c|}
\hline & $f_{B F 2 N}$ & $\lambda_{\max }(\mathrm{nm})$ & $\varepsilon\left(\mathrm{M}^{-1} \mathrm{~cm}^{-1}\right)$ & $\lambda_{\mathrm{em}}(\mathrm{nm})$ & $\Phi_{F}(\%)$ & $\mathrm{U}_{\mathrm{ST}}(\mathrm{nm})$ & $\mathrm{U}_{\mathrm{ST}}\left(\mathrm{cm}^{-1}\right)$ & $\mathrm{E}_{\text {red } 1}\left(\mathrm{~V}\right.$ vs $\left.\mathrm{Fc} / \mathrm{Fc}^{+}\right)$ & $\mathrm{E}_{\text {red2 }}\left(\mathrm{V}\right.$ vs. $\left.\mathrm{Fc} / \mathrm{Fc}^{+}\right)$ \\
\hline BF2N & 1 & 561 & 35,300 & 663 & 30 & 102 & 2742 & -0.71 & -1.75 \\
\hline PBF2N & 1 & 559 & 27,500 & 663 & 11 & 104 & 2806 & -0.71 & -1.75 \\
\hline \multirow[t]{3}{*}{$(\mathrm{PDND})_{\mathrm{m}}-r-(\mathrm{PBF} 2 \mathrm{~N})_{\mathrm{n}}$} & 0.50 & 558 & - & 664 & 8 & 106 & 2861 & -0.70 & -1.75 \\
\hline & 0.15 & 560 & - & 664 & 18 & 104 & 2797 & - & - \\
\hline & 0.08 & 561 & - & 664 & 24 & 103 & 2765 & - & - \\
\hline \multirow[t]{3}{*}{$(\text { PDND })_{m}-b-(\text { PBF2N })_{n}$} & 0.48 & 559 & - & 667 & 2 & 108 & 2897 & -0.70 & -1.75 \\
\hline & 0.13 & 559 & - & 665 & 3 & 106 & 2851 & - & - \\
\hline & 0.07 & 559 & - & 665 & 1 & 106 & 2851 & - & - \\
\hline
\end{tabular}



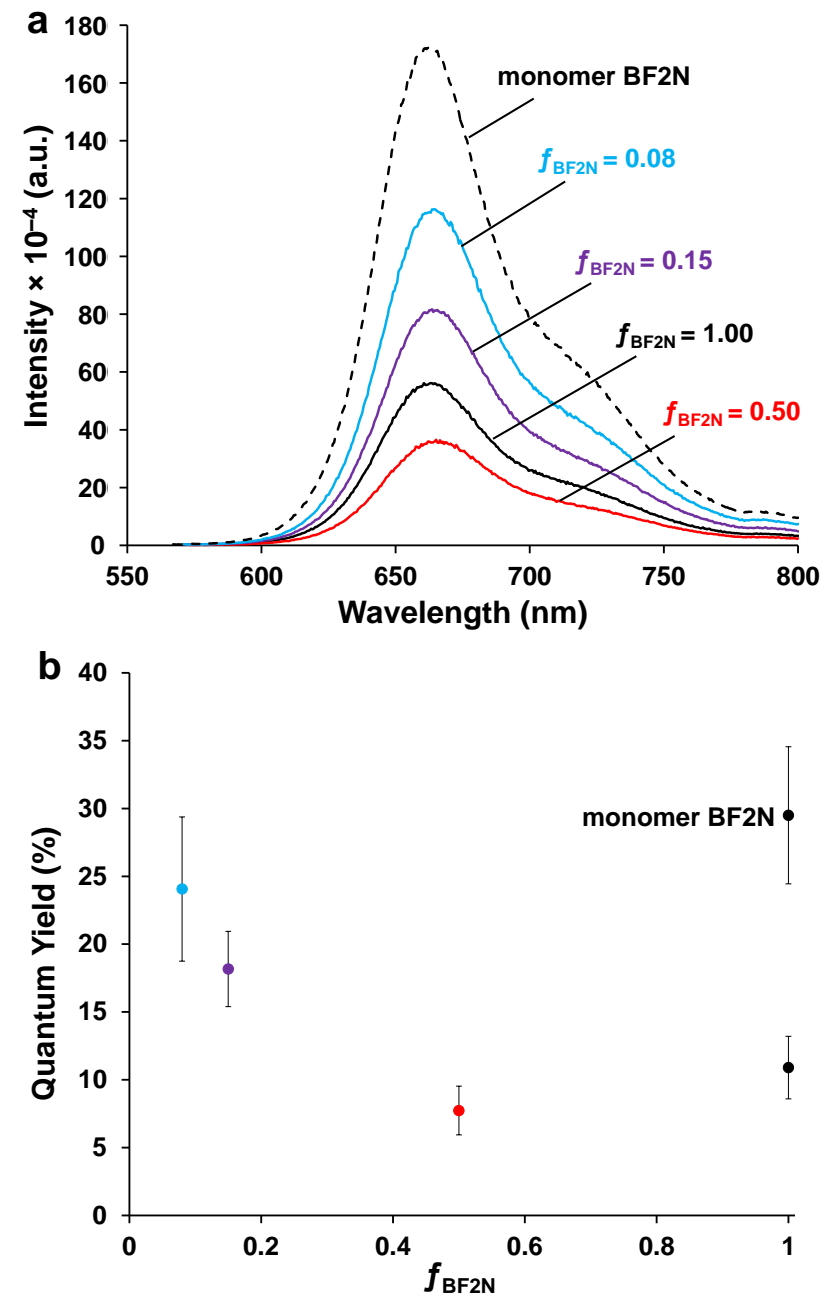

Fig. 3 (a) UV-vis emission spectra of monomer BF2N, homopolymer PBF2N, and random copolymers (PDND) m $^{-r}-($ PBF2N) $n$ recorded for degassed $\mathrm{CH}_{2} \mathrm{Cl}_{2}$ solutions with absorbances of 0.1 at the excitation wavelength of $560 \mathrm{~nm}$. (b) Quantum yields of the corresponding solutions. The error bars were calculated from a minimum of 3 independent experiments.

for this series of polymers were far more variable. The $\Phi_{F}$ estimated for monomer BF2N was 30\%, while that of homopolymer PBF2N was $11 \%$. We rationalize the decrease in $\Phi_{\mathrm{F}}$ based on the relatively close proximity of the BF2N units in the homopolymer, leading to increased reabsorption of emitted photons. The fact that $\Phi_{\mathrm{F}}$ decreased further when solution concentrations were increased supported this rationale. When the $f_{B F 2 N}$ was decreased in random copolymers (PDND) $)_{m}-r$-(PBF2N) $)_{n}$, quantum yields increased to a maximum value of $24 \%$ when $f_{B F 2 N}=0.08$. The random copolymerization of BF2N and DND effectively served to decrease the concentration of the $\mathbf{B F 2 N}$ repeat units in the polymer backbones, thereby attenuating reabsorption of emitted photons and increasing $\Phi_{\mathrm{F}}$. Block copolymers $(\text { PDND })_{m}-\boldsymbol{b}-(\text { PBF2N })_{n}$ were only weakly emissive $\left(\Phi_{\mathrm{F}}<3 \%\right)$, implying that solution-based aggregation may result in the BF2N units being forced into close proximity in solution.
However, we have been unable to observe nano-sized aggregates experimentally.

The cyclic voltammograms collected for monomer BF2N, homopolymer PBF2N, random copolymer (PDND) $)_{m}-r-($ PBF2N) $\left(f_{B F 2 N}=0.50\right)$, and block copolymer $(\text { PDND })_{m}-b-(P B F 2 N)_{n}\left(f_{B F 2 N}\right.$ $=0.48$ ) were each comprised of two reversible reduction waves centred at $c a$. $-0.71 \mathrm{~V}$ and $-1.75 \mathrm{~V}$ relative to the ferrocene/ferrocenium redox couple (Table 2, Fig. S18), as has been observed for other $\mathrm{BF}_{2}$ 3-cyanoformazante complexes. $^{32,36}$ The first reduction event corresponds to the conversion of the monomer/repeating units to ligand-centred radical anions and the second wave corresponds to their conversion to dianions.

\section{Conclusions}

ROMP was used to synthesize the first examples of homopolymers (PBF2N) and copolymers (PDND) $)_{m}-r-($ PBF2N) and (PDND) $)_{m}-\boldsymbol{b}-(\text { PBF2N })_{n}$ that contain pendant $\mathrm{BF}_{2}$ 3cyanoformazanate complexes. GPC and ${ }^{1} \mathrm{H}$ NMR spectroscopy were used to probe the molecular weight distributions of the polymers produced and to establish the $\mathrm{f}_{\mathrm{BF} 2 \mathrm{~N}}$ for each polymer. TGA and DSC revealed details of the thermal stability and each polymer and corroborated the proposed copolymer structures (i.e., random vs. block). Polymers containing BF2N repeating units retained many of the unique traits of molecular analogs, including: absorption maxima at $c a .560 \mathrm{~nm}$, emission maxima at $c a .665 \mathrm{~nm}$, and reversible electrochemical conversion to their radical anion and dianion forms. However, the origins of the observed trends in the intensity of the absorption and emission maxima in $\mathrm{CH}_{2} \mathrm{Cl}_{2}$ were not immediately clear. Through careful comparison of the spectra collected for monomer BF2N, homopolymer PBF2N, random copolymers $(\text { PDND })_{m}-r-(\text { PBF2N })_{n}$, and block copolymers (PDND) $)_{m}-b-$ $(\text { PBF2N })_{n}$ it was determined that the maximum absorption intensities were lower than expected when $f_{B F 2 N}$ was high due to an apparent masking effect caused by coiling of polymer chains in solution. Furthermore, by effectively diluting BF2N in the polymer chains, via random copolymerization, we were able to rejuvenate the emission associated with $\mathrm{BF}_{2} 3$ cyanoformazante complexes, and confirm that re-absorption of emitted photons was the likely mechanism of emission attenuation in homopolymer PBF2N, block copolymers $(\text { PDND })_{m}-b-(P B F 2 N)_{n}$, and random copolymers $(\text { PDND })_{m}-r-$ $(\text { PBF2N) })_{n}$ with relatively high $f_{B F 2 N}$. This work will ultimately serve as a guide for the design and synthesis of fluorescent polymers based on $\mathrm{BF}_{2}$ formazanate and related complexes with application as fluorescent materials in a variety of applications within the chemical biology and materials science communities.

\section{Conflict of interest}

There are no conflicts to declare. 


\section{Acknowledgements}

We would like to thank the University of Western Ontario, the Natural Science and Engineering Research Council (NSERC) of Canada (J. B. G.: DG, 435675; S. N.: CGS M scholarship), the Ontario Ministry of Research and Innovation (J. B. G.: ERA, ER14-10-147; S.N.: OGS scholarship) and the Canada Foundation for Innovation (J. B. G.: JELF, 33977) for funding this work. Finally we thank Prof. Elizabeth R. Gillies for access to instrumentation in her lab.

\section{Notes and references}

1 F. Jäkle, Chem. Rev., 2010, 110, 3985-4022.

2 F. Cheng and F. Jakle, Polym. Chem., 2011, 2, 2122-2132.

3 K. Tanaka and Y. Chujo, Macromol. Rapid Commun., 2012, 33, 1235-1255.

4 H. Helten, Chem. Eur. J., 2016, 22, 12972-12982.

5 C.-H. Zhao, A. Wakamiya and S. Yamaguchi, Macromolecules, 2007, 40, 3898-3900.

6 B. H. Lessard, K. L. Sampson, T. Plint and T. P. Bender, J. Polym. Sci., Part A: Polym. Chem., 2015, 53, 1996-2006.

7 S. M. Barbon and J. B. Gilroy, Polym. Chem., 2016, 7, 35893598.

8 X. Yin, F. Guo, R. A. Lalancette and F. Jäkle, Macromolecules, 2016, 49, 537-546.

9 T. Lorenz, M. Crumbach, T. Eckert, A. Lik and H. Helten, Angew. Chem. Int. Ed., 2017, 56, 2780-2784.

10 J. C. Sanchez and W. C. Trogler, J. Mater. Chem., 2008, 18, 5134-5141.

11 G. M. Pawar, R. A. Lalancette, E. M. Bonder, J. B. Sheridan and F. Jäkle, Macromolecules, 2015, 48, 6508-6515.

12 A. Hirose, K. Tanaka, R. Yoshii and Y. Chujo, Polym. Chem., 2015, 6, 5590-5595.

13 I. A. Adams and P. A. Rupar, Macromol. Rapid Commun., 2015, 36, 1336-1340.

14 V. M. Suresh, A. Bandyopadhyay, S. Roy, S. K. Pati and T. K. Maji, Chem. Eur. J., 2015, 21, 10799-10804.

15 Z. Lu, L. Mei, X. Zhang, Y. Wang, Y. Zhao and C. Li, Polym. Chem., 2013, 4, 5743-5750.

16 J. Qiao, Z. Liu, Y. Tian, M. Wu and Z. Niu, Chem. Commun., 2015, 51, 3641-3644.

17 C. Dai, D. Yang, X. Fu, Q. Chen, C. Zhu, Y. Cheng and L. Wang, Polym. Chem., 2015, 6, 5070-5076.

18 J. Chen, W. Zhong, Y. Tang, Z. Wu, Y. Li, P. Yi and J. Jiang, Macromolecules, 2015, 48, 3500-3508.

19 J. Winsberg, T. Hagemann, S. Muench, C. Friebe, B. Häupler, T. Janoschka, S. Morgenstern, M. D. Hager and U. S. Schubert, Chem. Mater., 2016, 28, 3401-3405.
20 B. Kim, B. W. Ma, V. R. Donuru, H. Liu and J. M. J. Fréchet, Chem. Commun., 2010, 46, 4148-4150.

21 S. Cataldo, S. Fabiano, F. Ferrante, F. Previti, S. Patanè and B. Pignataro, Macromol. Rapid Commun., 2010, 31, 1281-1286.

22 S. P. Economopoulos, C. L. Chochos, H. A. Ioannidou, M. Neophytou, C. Charilaou, G. A. Zissimou, J. M. Frost, T. Sachetan, M. Shahid, J. Nelson, M. Heeney, D. D. C. Bradley, G. Itskos, P. A. Koutentis and S. A. Choulis, RSC Adv., 2013, 3, 10221-10229.

23 C. Dou, X. Long, Z. Ding, Z. Xie, J. Liu and L. Wang, Angew. Chem. Int. Ed., 2016, 55, 1436-1440.

24 G. Zhang, G. M. Palmer, M. W. Dewhirst and C. L. Fraser, Nat. Mater., 2009, 8, 747-751.

25 A. Nagai, K. Kokado, J. Miyake and Y. Chujo, Macromolecules, 2009, 42, 5446-5452.

26 Z. M. Hudson, D. J. Lunn, M. A. Winnik and I. Manners, Nat. Commun., 2014, 5, 3372.

27 H. Qiu, Z. M. Hudson, M. A. Winnik and I. Manners, Science, 2015, 347, 1329-1332.

28 H. Qiu, Y. Gao, C. E. Boott, O. E. C. Gould, R. L. Harniman, M. J. Miles, S. E. D. Webb, M. A. Winnik and I. Manners, Science, 2016, 352, 697-701.

29 S. Novoa, J. A. Paquette, S. M. Barbon, R. R. Maar and J. B. Gilroy, J. Mater. Chem. C, 2016, 4, 3987-3994.

30 M.-C. Chang and E. Otten, Chem. Commun., 2014, 50, 74317433.

31 S. M. Barbon, J. T. Price, P. A. Reinkeluers and J. B. Gilroy, Inorg. Chem., 2014, 53, 10585-10593.

32 S. M. Barbon, P. A. Reinkeluers, J. T. Price, V. N. Staroverov and J. B. Gilroy, Chem. Eur. J., 2014, 20, 11340-11344.

33 R. R. Maar, S. M. Barbon, N. Sharma, H. Groom, L. G. Luyt and J. B. Gilroy, Chem. Eur. J., 2015, 21, 15589-15599.

34 R. R. Maar and J. B. Gilroy, J. Mater. Chem. C, 2016, 4, 64786482.

35 J. A. Paquette, A. Rabiee Kenaree and J. B. Gilroy, Polym. Chem., 2017, 8, 2164-2172.

36 S. M. Barbon, S. Novoa, D. Bender, H. Groom, L. G. Luyt and J. B. Gilroy, Org. Chem. Front., 2017, 178-190.

37 A. D. Hennis, J. D. Polley, G. S. Long, A. Sen, D. Yandulov, J. Lipian, G. M. Benedikt, L. F. Rhodes and J. Huffman, Organometallics, 2001, 20, 2802-2812.

38 J. A. Love, J. P. Morgan, T. M. Trnka and R. H. Grubbs, Angew. Chem. Int. Ed., 2002, 41, 4035-4037.

39 S. Fery-Forgues and D. Lavabre, J. Chem. Educ., 1999, 76, 1260.

40 K. Suzuki, A. Kobayashi, S. Kaneko, K. Takehira, T. Yoshihara, H. Ishida, Y. Shiina, S. Oishi and S. Tobita, Phys. Chem. Chem. Phys., 2009, 11, 9850-9860. 